\title{
Prosthetic knee joint infection due to Listeria monocytogenes bacteremia in a diabetic female
}

\author{
Yun Seo, Yong-sun Noh, Seong-Heon Wie, and U-Im Chang
}

Department of Internal Medicine, College of Medicine, The Catholic University of Korea, Seoul, Korea

Received: November 23, 2014

Revised : December 5, 2014

Accepted: December 9, 2014

\section{Correspondence to}

U-Im Chang, M.D.

Department of Internal Medicine, College of Medicine, St. Vincent's Hospital, The Catholic University of Korea, 93 Jungbu-daero, Paldal-gu, Suwon 16247, Korea

Tel: $+82-31-249-8313$

Fax: +82-31-253-8898

E-mail: cui7o@catholic.ac.kr

\section{To the Editor,}

Listeria monocytogenes is an opportunistic pathogen that can cause three types of systemic infection: isolated bacteremia, central nervous system infection, and maternal-fetal infection. These infections primarily occur in high-risk groups including neonates, pregnant women, elderly, and immunocompromised patients, with mortality rates of $20 \%$ to $40 \%$. Although rarely involved in prosthetic joint infection (PJI) or periprosthetic joint infection, L. monocytogenes has recently become a topic of interest due to the increase in prosthetic joint replacements in immunocompromised patients $[1,2]$. Since 1973 , there have been 54 cases of listeriosis in Korea; however, none of these cases reported PJI or periprosthetic joint infection. The management of periprosthetic joint infection or PJI almost always requires surgical intervention and prolonged courses of intravenous or oral antimicrobial therapy [3]. Appropriate antibiotic treatment during the early stages of PJI is an important factor for a good outcome. In this study, we report a 61-year-old diabetic female with a prosthetic knee joint infection associated with L. monocytogenes bacteremia. In this case, the patient had been receiving steroid therapy and has iatrogenic Cushing syndrome. This is an important case considering the high mortality rate associated with listeriosis and its rarity in being associated with PJIs, especially in Korea.

A 61-year-old female with diabetes mellitus (DM) and iatrogenic Cushing syndrome was admitted to the hospital because of fever, abdominal pain, and diarrhea on October 18, 2014. The patient was well, until fever and diarrhea occurred 2 days prior to presentation. She was diagnosed with DM and iatrogenic Cushing syndrome 8 years prior to presentation. The patient's iatrogenic Cushing syndrome was caused by ingestion of glucocorticoids and steroid injections, which had been taken for several years to relieve knee pain. DM was treated with a split-mixed insulin regimen taken twice daily. To treat iatrogenic Cushing syndrome, the patient was prescribed $5 \mathrm{mg}$ of prednisolone and $0.1 \mathrm{mg}$ of fludrocortisone, taken daily. Because of osteoarthritis, she had a history of total knee replacement arthroplasty of the left knee on January 11, 2008, and of the right knee on August 8,2012 . She did not drink alcohol or smoke tobacco, and had not come into contact with any febrile persons or anyone with symptoms similar to her; furthermore, she had not travelled to a foreign country in the past 10 years. Physical examination revealed mild tenderness around the area of the left knee joint. Examination revealed the following: blood pressure of $135 / 90 \mathrm{mmHg}$, pulse rate of 96 beats per minute, respi- 


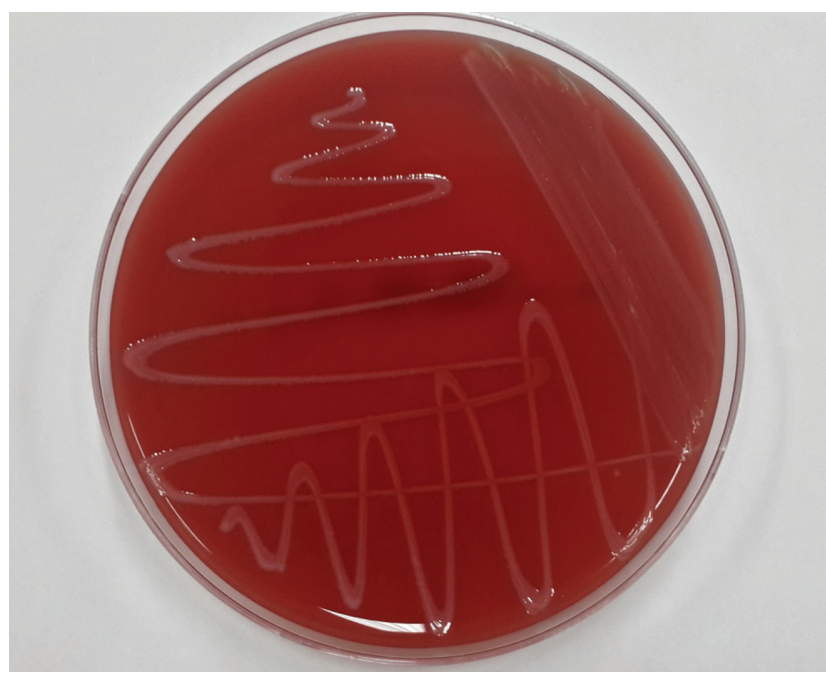

Figure 1. Beta-hemolytic and semi-transparent Listeria monocytogenes colonies grown on blood agar for 24 hours.

ratory rate of 22 breaths per minute, and a temperature of $39.0^{\circ} \mathrm{C}$. She also had a Cushingoid appearance, although examination of both knees and a neurological examination were normal.

Laboratory investigation revealed the following: white blood cell count of $22,170 / \mathrm{mm}^{3}$ (88.6\% neutrophils, $5.5 \%$ lymphocytes, and 5.9\% monocytes), hemoglobin level of $12.6 \mathrm{~g} / \mathrm{dL}$, platelet count of $191,000 / \mathrm{mm}^{3}$, C-reactive protein of $34.47 \mathrm{mg} / \mathrm{dL}$, blood urea nitrogen of $26.4 \mathrm{mg} / \mathrm{dL}$, and a creatinine level of $1.1 \mathrm{mg} / \mathrm{dL}$. Electrolytes, calcium, phosphorus, and liver enzyme levels were within normal limits. Urinalysis indicated 1 to 4 white blood cells and o to 1 red blood cells per high power field. Chest radiographs showed minimal bronchiectasis with linear atelectasis at the posterior basal segment of both lower lobes. A computerized tomography scan of the abdomen and pelvis showed nonspecific findings.

After performing peripheral blood and urine cultures, $2 \mathrm{~g}$ of ceftriaxone was administered intravenously twice daily. Although the standard dose of ceftriaxone is $2 \mathrm{~g}$ per 24 hours, the dosage can be increased for serious infections to a maximum of $4 \mathrm{~g}$ per 24 hours, if divided and given every 12 hours. The patient was considered immunocompromised at initial presentation for underlying DM and her long-term use of glucocorticoids. Therefore, we initiated empirical antimicrobial therapy with broad-spectrum ceftriaxone at a maximum dose of $2 \mathrm{~g}$ per 12 hours to prevent the development of bactere-

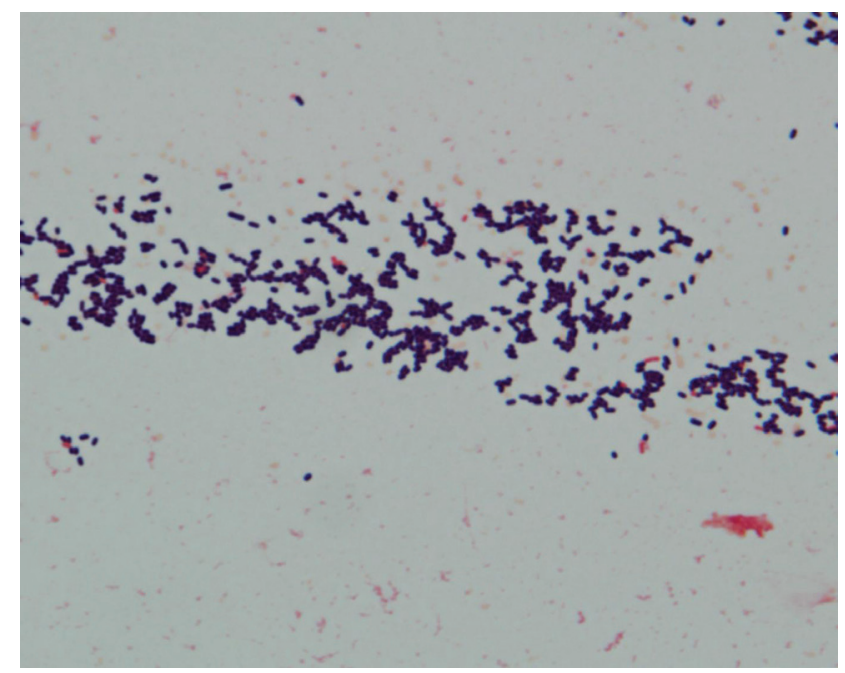

Figure 2. Gram stain of a blood culture indicates the presence of gram-positive coccobacilli (light microscope at $\times 1,000$ magnification).

mia. During the first 2 days of hospitalization, the patient only complained of persistent diarrhea (on average, five episodes per day). On the third day of hospitalization, initial antibiotic regimen was changed to $4.5 \mathrm{~g}$ of intravenous piperacillin-tazobactam every 8 hours, since fever did not subside. On the morning of the third day, the patient complained of sudden left knee pain which became aggravated as she tried to walk. On examination, there was redness and swelling with extreme tenderness of the left knee, which had not manifested on the first day of hospitalization. Anteroposterior radiographs of the left knee were unremarkable. As a PJI was suspected, ultrasound-guided joint fluid aspiration of the left knee was performed and thick, pus-like synovial effusion was present. Laboratory tests of the synovial fluid revealed the following: white blood cell counts of 27,300/ $\mathrm{mm}^{3}$ (79.0\% neutrophils, 6.0\% lymphocytes, and 15.0\% monocytes) and red blood cell counts of $8,000 / \mathrm{mm}^{3}$. Synovial fluid analysis raised suspicion of septic arthritis of the left knee. Meanwhile, L. monocytogenes was recovered from initial and follow-up blood cultures on the third day of hospitalization (Figs. 1 and 2). Antibiotic therapy was changed to $2 \mathrm{~g}$ of intravenous ampicillin every 4 hours plus $160 \mathrm{mg}$ intravenous trimethoprim/800 mg sulfamethoxazole every 12 hours.

Surgical exploration of the left knee was performed on October 23, 2014 (day 6 of hospitalization), which was 3 days after the onset of left knee pain. A pus-like effusion 
released at the site of the incision on the left knee. Infected granulation tissue and synovial hypertrophy were identified and debridement of the tissue with total synovectomy was performed. Surgical findings were consistent with PJI. Since prostheses were well-fixed without a sinus tract and the time from the onset of symptoms to the operation was less than a few weeks, prostheses were retained. Synovial fluid and granulation tissue cultures tested negative. On day 5 of hospitalization, the patient's fever subsided and her condition was stable. On day 8 , follow-up blood cultures tested negative. Intravenous ampicillin and trimethoprim-sulfamethoxazole were discontinued after 6 weeks. The patient was diacharged and prescribed $500 \mathrm{mg}$ oral amoxicillin/125 mg clavulanate and $160 \mathrm{mg}$ oral trimethoprim/80o mg sulfamethoxazole every 12 hours, which was continued for a total of 7 weeks. On week 7 of follow-up, the patient was symptomless. However, we decided to perform a maintenance regimen by using oral antibiotics and weekly or bimonthly follow-up investigations for the next 5 weeks. Finally, we reassessed the patient's condition after 12 weeks of antibiotic treatment since the median duration of antibiotic treatment was 15 weeks (range, 2 to 88 ) in other reported cases of L. monocytogenes-associated joint and bone infections [1].

L. monocytogenes is an opportunistic pathogen affecting immunocompromised patients. Resistance to $L$. monocytogenes infection is predominantly cell-mediated, which can be observed by the overwhelming clinical association between listeriosis and conditions associated with impaired cellular immunity including lymphomas, pregnancy, acquired immune deficiency syndrome, and corticosteroid-induced immunosuppression [1]. According to a French study of 43 cases of L. monocytogenes-associated joint and bone infections, putative risk factors for bone and joint listeriosis appeared to be a combination of those identified for bacterial arthritis and listeriosis with the following being the major contributors: (1) age $>60$ years, (2) presence of foreign material, (3) immunosuppression and corticosteroid therapy, (4) underlying neoplasia, and (5) diabetes [1]. The patient in this study had three of the five abovementioned risk factors (i.e., age, corticosteroid therapy, and diabetes), which are presumed to have led to decreased cellular immunity and listerial gastroenteritis, eventually resulting in bacteremia and PJI.
In the United States, the overall incidence of listeriosis is approximately 7.9 cases per million population. However, after active surveillance and prevention efforts by the Centers for Disease Control and Prevention, the incidence rate of listeriosis declined from 7.9 per 1 million in 1989 to 4.4 per 1 million in 1993 [2]. In Europe, the overall incidence of listeriosis is estimated to be approximately 4.7 cases per million population. A French study reviewed 43 cases of articular listeriosis that had been reported to the French National Reference Center for Listeriosis between 1992 and 2010. Among these 43 cases of articular listeriosis, 38 patients (88\%) were $\geq 60$ years, 36 patients $(84 \%)$ had prosthetic joints, 14 patients (33\%) had a history of use of immunosuppressive agents within 5 years, 11 patients (26\%) had neoplasms, seven patients (16\%) had rheumatoid arthritis, and five patients (11\%) had diabetes [1].

According to the Infectious Diseases Society of America (IDSA) Clinical Practice Guidelines of Diagnosis and Management of PJI, the case in this study was defined as PJI because the patient had purulence in the affected joint [3]. The possibility of PJI might have been more conclusive if the pre- and intraoperative cultures of synovial fluid and granulation tissues tested positive. However, synovial fluid cultures were negative. Although we observed a negative culture result, there has been a low incidence (o\% to $25 \%$ ) of cases in which cultures have tested positive for L. monocytogenes in PJIs. The results observed in this study might be explained by a study performed by Choi et al. [4] that investigated negative culture results and the association with periprosthetic joint infections. However, the negative results could also be due to the patient's prior use of antibiotics, since we had administered ceftriaxone and piperacillin-tazobactam prior to surgery.

Management of PJI consists of surgical intervention and prolonged courses of antimicrobial therapy. Based on the condition of PJI, different surgical strategies are applied. According to IDSA Clinical Practice Guidelines of PJI, patients diagnosed with PJI that have a well-fixed prosthesis without a sinus tract, are within 30 days of prosthesis implantation, or are less than 3 weeks from the onset of infectious symptoms, should be considered for debridement and retention of their prostheses [3]. In this study, the patient satisfied all of the above conditions. Therefore, we performed debridement of infected granulation tissue with total synovectomy.

At present, there are no guidelines on the necessary 
therapeutic regimen for local L. monocytogenes infections. Treatment for listerial infections of prosthetics is experimental because these infections are rare. In general, the accepted therapy consists of a combination of ampicillin and gentamicin. In cases in which an individual is allergic to ampicillin, trimethoprim-sulfamethoxazole can be utilized [5]. Since ampicillin is not bactericidal against L. monocytogenes, use in combination with a bactericidal agent is important in the treatment of PJIs. In this study, the patient's initial and follow-up serum creatinine levels were between 1.1 to $1.3 \mathrm{mg} / \mathrm{dL}$. Considering nephrotoxicity of aminoglycosides, we prescribed the patient a combination regimen of ampicillin and trimethoprim-sulfamethoxazole. A previous study showed that a combination of ampicillin and trimethoprim-sulfamethoxazole could be used to effectively treat severe listerial meningoencephalitis [5].

According to the IDSA guidelines, 4 to 6 weeks of pathogen-specific intravenous or oral antimicrobial therapy is necessary to effectively treat PJIs caused by organisms other than staphylococci [3]. However, antibiotics have been prescribed for up to 3 months. Considering the high mortality rate associated with listeriosis and its rarity in PJIs, the case reported in this study, of a PJI associated with L. monocytogenes in Korea, is of clinical significance. Our study suggests that prosthetic knee joint infections associated with L. monocytogenes should be considered in the differential diagnosis of septic arthritis in patients who have been administered immunosuppressants.
Keywords: Listeria monocytogenes; Bacteremia; Arthritis

\section{Conflict of interest}

No potential conflict of interest relevant to this article was reported.

\section{REFERENCES}

1. Charlier C, Leclercq A, Cazenave B, et al. Listeria monocytogenes-associated joint and bone infections: a study of 43 consecutive cases. Clin Infect Dis 2012;54:240-248.

2. Tappero JW, Schuchat A, Deaver KA, Mascola L, Wenger JD. Reduction in the incidence of human listeriosis in the United States: effectiveness of prevention efforts? The Listeriosis Study Group. JAMA 1995;273:1118-1122.

3. Osmon DR, Berbari EF, Berendt AR, et al. Diagnosis and management of prosthetic joint infection: clinical practice guidelines by the Infectious Diseases Society of America. Clin Infect Dis 2013;56:e1-e25.

4. Choi HR, Kwon YM, Freiberg AA, Nelson SB, Malchau H. Periprosthetic joint infection with negative culture results: clinical characteristics and treatment outcome. J Arthroplasty 2013;28:899-903.

5. Merle-Melet M, Dossou-Gbete L, Maurer P, et al. Is amoxicillin-cotrimoxazole the most appropriate antibiotic regimen for listeria meningoencephalitis? Review of 22 cases and the literature. J Infect 1996;33:79-85. 